Cinémas

Revue d'études cinématographiques

Journal of Film Studies

\title{
Le dernier des discrets
}

\section{The Last Modest Scholar}

\section{Serge Cardinal}

Volume 19, numéro 1, automne 2008

Les transformations du cinéma

URI : https://id.erudit.org/iderudit/029501ar

DOI : https://doi.org/10.7202/029501ar

Aller au sommaire du numéro

Éditeur(s)

Cinémas

ISSN

1181-6945 (imprimé)

1705-6500 (numérique)

Découvrir la revue

Citer cet article

Cardinal, S. (2008). Le dernier des discrets. Cinémas, 19(1), 109-126.

https://doi.org/10.7202/029501ar
Résumé de l'article

Cofondateur de la revue Cinémas, Michel Larouche a enseigné au Département d'histoire de l'art et d'études cinématographiques de l'Université de Montréal jusqu'en 2005. Ses travaux nous ont fait découvrir des cinémas méconnus (le cinéma expérimental ou d'Afrique), des cinémas ignorés (celui de Jodorowsky ou le cinéma indépendant québécois), des cinémas hybrides (ceux qui s'approprient la photographie, les nouvelles technologies ou la littérature). Mais quelle méthode, quels problèmes et quels concepts Michel Larouche nous laisse-t-il en héritage ? Surtout, quelle éthique de la pensée a-t-il discrètement laissée aux études cinématographiques? 


\title{
Le dernier des discrets
}

\section{Serge Cardinal}

\begin{abstract}
RÉSUMÉ
Cofondateur de la revue Cinémas, Michel Larouche a enseigné au Département d'histoire de l'art et d'études cinématographiques de l'Université de Montréal jusqu'en 2005. Ses travaux nous ont fait découvrir des cinémas méconnus (le cinéma expérimental ou d'Afrique), des cinémas ignorés (celui de Jodorowsky ou le cinéma indépendant québécois), des cinémas hybrides (ceux qui s'approprient la photographie, les nouvelles technologies ou la littérature). Mais quelle méthode, quels problèmes et quels concepts Michel Larouche nous laisse-t-il en héritage? Surtout, quelle éthique de la pensée a-t-il discrètement laissée aux études cinématographiques?
\end{abstract}

For English abstract, see end of article

Faire le portrait conceptuel de Michel Larouche: tracer une image de sa pensée, reposer ses problèmes, recomposer ses concepts, reconstituer ses mouvements descriptifs, analytiques ou argumentatifs, et ce, de sorte que cette matière, ces traits et ces trajets idéels capturent, conservent et reproduisent une image de l'homme, sa posture, son timbre, son rythme. Un portrait à double face: les problèmes qui l'occupent, les concepts qu'il taille, les mouvements que la conceptualisation et la problématisation lui imposent, seraient autant de signes d'une manière de vivre qui s'inventait à travers lui. Nous avons toujours les problèmes, les concepts, la méthode que nous méritons en fonction de notre manière d'être ou de notre style de vie. Le sens d'un concept, c'est sa capacité à composer les variables et les variations d'un problème qui s'est emparé de l'image ou de la théorie de l'image; la valeur de ce problème, c'est sa puissance de sélection de l'important et de l'intéressant, sa puissance de répartition du singulier et du régulier, du remarquable et de l'ordinaire; cette valeur suppose un point de vue d'évaluation: une manière d'être, un mode d'existence, un style de vie. Non pas 
notre histoire personnelle, nos goûts ou nos opinions, nos fantasmes ou nos traumatismes, mais, d'une part, nos mouvements cinétiques, nos rapports de vitesse et de lenteur, et, d'autre part, nos mouvements dynamiques, nos capacités d'affecter et d'être affecté, nos seuils de tolérance, nos types d'angoisse ou de sérénité. Par exemple, quel régime répétitif du regard et quelle angoisse du temps faut-il à Michel Larouche pour être ainsi fasciné par l'immobilisation d'une image de cinéma? Fascination telle qu'il doit en dégager un problème esthétique et éthique, qu'il doit composer ce concept d'effet-photo désignant la genèse de la perception et du sens, qu'il doit inventer un mode d'existence capable de transformer la mélancolie photographique en une renaissance à soi. L'important, ce n'est pas la photo de sa mère, encore qu'il la gardât peut-être précieusement dans son portefeuille, ni son savoir avéré de l'expérimentation filmique, mais sa manière d'être au temps et à l'espace qui transformait un objet de connaissance ou une curiosité esthétique en un véritable problème pour la sensibilité et la mémoire. Il faut remonter jusque-là si l'on espère retrouver le sens des concepts et la valeur des problèmes, leur force et leur puissance. Qu'est-ce qu'il veut, celui qui dit: "Tous les arts sont fondés sur la présence de l'homme; dans la seule photographie nous jouissons de son absence» (Bazin 1985, p. 13)? Qu'est-ce qu'il veut, celui qui dit: "L'effet photographie ouvre à un hors champ subtil [...] qui génère non pas des émotions de masse (la peur, le rire, les larmes), mais des émotions nouvelles, plus difficilement identifiables» (Larouche 1988, p. 136)?

Il faudrait que le portrait ait la ressemblance du double: il rappellerait à ma mémoire ce que je n'ai ni vu ni lu, ce que Michel Larouche faisait discrètement entendre, que ce soit en ma présence ou sur la page, mais que mon bavardage de collégien ou mon empressement de publicitaire m'empêchaient de voir ou de lire. Un théoricien laisse sans doute en héritage ses objets, ses problèmes, ses concepts, sa méthode. C'est ce dont on se souvient parce qu'ils se présentaient sous leur face immédiatement utilisable: tel objet pour tel colloque, tel problème pour telle note de bas de page, tel concept pour telle fin de paragraphe. À cet héritage, on peut répondre en prenant l'encensoir 
ou à coups d'objections, en reprenant ou en réfutant: avec Michel Larouche, on continuera à faire l'histoire du cinéma expérimental, dont on retrouvera les problèmes et les questions dans les pratiques vidéographiques et numériques; après lui, on s'intéressera à la réception des films québécois, mais pour en dégager des principes de marketing; contre lui, on objectera que l'adaptation est affaire de transmutation et non de transcodage, etc. Mais le théoricien nous laisse aussi en héritage sa manière d'être: un style de répétition, un mode temporel, par exemple. C'est ce dont on se souvient sans jamais l'avoir vraiment vu ou lu parce que c'était, et ça reste, inutilisable: c'est ce qui donne un sens aux concepts et une valeur aux problèmes. Et cela, on ne peut se contenter de le reprendre ou de le poursuivre; et à cela, il n'y a rien à objecter. Car cette manière d'être, paradoxalement, c'est elle qui rend possible, et quelquefois nécessaire, la plus grave objection, la plus profonde répétition, la plus folle poursuite, celles qui vous font remonter jusqu'à vos propres conditions d'existence et de pensée. On pourra alors conserver le même mot, effet-photo par exemple, ou le changer, mais cela n'aura plus d'importance parce que, tout en désignant les mêmes images immobilisées, il renverra à un tout autre regard et à une tout autre affection temporelle, à un mode d'existence en train de s'inventer. Et là commence sans doute le plus bel héritage du théoricien, quand il ne provoque plus seulement des ajustements, des adaptations, des objections, la relativité et la rivalité des vérités d'un même monde, mais quand il oblige à inventer des mondes, de nouveaux paradigmes, de nouvelles finalités, etc., "plus difficilement identifiables".

Tout commence dans la pluralité. Dès les premières pages de son premier grand texte, Michel Larouche veut rendre compte de la pluralité constitutive de ces étranges objets que sont les films, et répondre à cette pluralité en élevant l'emprunt, le bouturage et la bifurcation au rang de méthode: il veut "mettre en valeur la complexité et la richesse que recèlent les films»; il n'y parviendra qu'en utilisant «au maximum la pertinence de 
diverses méthodes" (Larouche 1985b, p. 11-12). Le théoricien s'engage dans une affaire difficile parce que se pose immédiatement la double question de la consistance et de la cohérence du discours: qu'est-ce qui donne une consistance à l'hétérogénéité du discours filmique? Qu'est-ce qui donne une cohérence à l'interdisciplinarité de la méthode? À une telle question on peut toujours répondre par des précautions méthodologiques et épistémologiques; on le verra, la réponse de Michel Larouche aura la complexité d'une posture éthique. Pour l'instant, on doit se contenter de découvrir certains des choix méthodologiques qui découlent d'une telle posture éthique. D'une part, Michel Larouche refuse de s'intéresser à un texte filmique refermé sur lui-même, replié dans sa logique propre: un film ne tient jamais tout seul. D'autre part, il ignore la possibilité même de travailler sur plusieurs textes filmiques, se gardant de suivre le fil d'un illusoire déterminisme de proche en proche entre des œuvres qui se succèdent suivant l'ordre de leur création, se gardant de retrouver leur régime de cohérence dans un thème caché commun ou une procédure unique: les films ne tiennent pas ensemble. Ils ne tiennent ni comme tout ni comme parties d'un tout, parce qu'ils font série et sont eux-mêmes sériels: par deux, par trois, par mille, ou chacun par soi-même, il faut les considérer "comme un vaste texte plurifilmique" (Larouche 1985b, p. 12). Les films de Jodorowsky, mais tout aussi bien les scénarios et les adaptations de l'œuvre de Gabrielle Roy (Larouche 1997 b et 1997c) ou encore les films photographiques (1995d), sont des "éléments d'un même discours fondamental" (1985b, p. 34) ou d'un seul texte pluriel, traversé par un axe d'orientation qui intègre les différents systèmes signifiants: système interculturel, système intermédiatique, système mythique, dramaturgique, etc. (p. 12).

On dira qu'on exagère, qu'on lit mal: "un même discours fondamental ", un axe d'orientation intégrateur, n'est-ce pas précisément dans cette unité et cette homogénéité que la pluralité vient trouver sa dernière cohérence et son ultime consistance? Mais ne voit-on pas qu'il s'agit d'une manière de parler l'idiome théorique de l'époque, de ne pas attirer l'attention sur soi, tandis qu'on s'intéresse déjà discrètement à autre chose. 
Michel Larouche est un grand comique. Bien sûr, il dit "axe d'orientation", mais cet axe, a-t-on bien compris à quelle géométrie il appartenait? Peut-on imaginer qu'un admirateur du film Région centrale, de Michael Snow, en soit encore à une géométrie euclidienne ou projective? Comment ne pas voir qu'il est déjà embarqué, même intuitivement, surtout intuitivement, dans une géométrie différentielle? Sur ce plan, un point de vue ou un axe d'orientation équivaut à la série des transformations qu'on fait subir aux propriétés intrinsèques d'une figure, à partir d'une position dans cette figure, c'est-à-dire en ne sortant pas de son espace, ce qui élimine toute référence à un espace plus grand qui la contiendrait. C'est pourquoi, quand Michel Larouche cherche à découvrir, dans tel ou tel texte plurifilmique, cet axe d'orientation, il trouve d'abord une série de bifurcations. Ainsi de son analyse d'India Song, de Marguerite Duras. Bien sûr, il retient du film trois choses évidentes: indifférente, voire «impitoyable», la musique se fait entendre "malgré » l'action qui se déroule à l'écran; les voix ne trouvent jamais de corps où venir s'ancrer, les dialogues eux-mêmes restent flottants; les corps gravitent autour d'un personnage "qui prête son apparence à un portrait de femme morte» (Larouche 1985a, p. 68). Mais s'il s'en tient prudemment aux évidences, c'est pour les jouer sur l'improbable: l'indifférence de la musique, le flottement des voix, la solitude des corps, vont constituer en soi l'" axe d'orientation" du film. Mais quel axe, au juste? Un axe doublement orienté, suivant des directions contradictoires et pourtant coexistantes: "la destruction d'un amour par la mort en même temps que son prolongement par la mémoire» (p. 68). Quand il cherche à découvrir cet axe d'orientation, Michel Larouche trouve ensuite un élément circulant d'un segment, d'un fragment, d'un niveau ou d'un système à l'autre, élément polymorphe et polysémique. S’agissant de l'œuvre de Jodorowsky, il part à la recherche du "système des systèmes »: ce qui intègre tous les systèmes - système culturel, générique, chromatique et musical, système des personnages —, n'est-ce pas ultimement le système mythique? En apparence, peut-être, mais plus profondément, c'est un élément appartenant à l'un des systèmes, le théâtre, qui va prendre sur lui l'opération de consistance et de 
cohérence: le masque. Le masque est "un motif complexe chargé de sens à partir duquel on peut ramener toute la constellation jodorowskienne" (Larouche 1985b, p. 170). S’il est "complexe", c'est qu'il n'est que métamorphose: tantôt, ce sont les stéréotypes du western qui prennent l'apparence de masques; tantôt, c'est l'acteur qui est un porteur de masque; tantôt, c'est le masque qui sert de médiateur entre l'homme, la nature et le surnaturel. Et s'il est "chargé " de sens, c'est qu'en étant mensonge et vérité, ludique et sacré, théâtral et mythique, analogique et symbolique, il peut attirer à lui les contraires et les différences: le masque n'étant que métamorphose, il permet d'agencer les déséquilibres et les hétérogénéités en un champ de tensions (Larouche 1985b, p. 170-171). Cet élément polymorphe et polysémique, qui donne consistance et cohérence en circulant, on le retrouve encore dans l'écriture critique: c'est «le trajet délibéré d'un point de vue» qui donne au texte critique une force argumentative, narrative et poétique, une capacité de reproduire la cinétique et la dynamique du film analysé ou décrit, au point de rivaliser avec lui, à condition que ce point de vue soit lui-même passage d'un style, d'un mode ou d'une voix à l'autre, comme en témoignent exemplairement les textes du critique Patrick Straram, que Michel Larouche aime à citer (Larouche et Cardinal 1996b, p. 134-136). Cet élément, on le retrouve enfin dans le passage d'un système sémiotique à l'autre. Par exemple, l'adaptation filmique d'une nouvelle de Gabrielle Roy, «Le vieillard et l'enfant», qui a beaucoup intéressé Michel Larouche, ne réussit la "transmédiatisation» obligée qu'en laissant le dédoublement de la voix narrative circuler, tout en se transformant, du texte littéraire au texte filmique: la voix off, « racontant à la première personne du singulier en utilisant l'imparfait et le passé simple", "constitue une analogie cinématographique de ce double palier inscrit dans la nouvelle, fondé sur les commentaires de la narratrice enchâssant la naïveté de la fillette dans une réflexion avantageusement décalée» (Larouche 1997b, p. 11-12).

Cette pluralité du texte filmique, Michel Larouche va donc l'aborder et l'explorer suivant une méthode elle-même plurielle: plutôt que de chercher "à appliquer une grille d'analyse 
unique", il va utiliser "au maximum la pertinence de diverses méthodes" (1985b, p. 12). Plutôt que d'adopter la perspective d'une analyse théorique, qui recherche en tout film la manifestation d'une structure abstraite parce qu'elle vise à s'élever du récit particulier à une théorie du récit, ou d'une figure particulière à une rhétorique générale, etc., Michel Larouche va adopter le point de vue de l'analyse descriptive qui, s'attachant à comprendre un texte concret, mesure son efficacité non pas à son pouvoir d'abstraction ou de généralisation, mais à sa capacité de répétition et de variation, multipliant s'il le faut les méthodes pour que ce texte traverse la série des points de singularité qu'il implique (points de rencontre, de rupture, d'hybridité, de transcodage, etc.). S’agissant du cinéma de Jodorowsky, par exemple, il recourt à la grande syntagmatique de Metz pour montrer que les films El Topo et La Montagne sacrée n'imitent pas le cinéma classique sans le détourner, puis à l'analyse intertextuelle pour montrer qu'ils ne détournent pas le cinéma classique sans reprendre et renverser le western et enfin à l'analyse intermédiatique pour montrer que ce détournement, cette reprise et ce renversement impliquent le recyclage de formes théâtrales et de structures musicales (Larouche 1985b). L'analyse descriptive qu'il pratique est donc répétitive et multiplicatrice. S'il en est ainsi, c'est que le texte concret qu'il s'attache à comprendre n'est jamais un texte particulier; c'est un texte singulier : une combinaison unique de systèmes hétérogènes, une mutation obtenue grâce à l'amalgame de différents genres (le cinéma de Jodorowsky ou le cinéma expérimental québécois), au transfert d'éléments d'un régime sémiotique à un autre (l'adaptation), au passage d'un régime de perception à un autre (le cinéma de l'affect). Si, pour Michel Larouche, le texte filmique est pluriel, plus profondément il est différentiel. D'où la nécessité pour le théoricien de disposer de plusieurs approches, chacune capable de saisir l'un des niveaux du texte comme une répétition et une variation de tous ses éléments. D'où également le fait qu'il lui faut porter jusqu'à son "maximum» la pertinence de chacune des méthodes, c'est-à-dire précisément porter chacune à sa limite. Chaque méthode employée a le pouvoir de pénétrer, d'organiser, de révéler et plus encore de construire le texte, 
efficacité qui se double d'une efficacité supérieure: en raison des insuffisances, des limites, des points aveugles de la méthode, voilà que le texte peut lui répondre dans son propre langage, faire penser l'analyse plutôt que d'être expliqué par elle. C'est quand l'écriture de la critique, par exemple, atteint sa limite analytique qu'elle "développe une énergie "désespérée" à pousser l'écriture jusqu'aux confins de sa capacité à "maîtriser" l'image et, ce faisant, déploie toute la complexité du récit littéraire, exhibe la problématique de sa représentation» (Larouche et Cardinal 1996b, p. 137-138). Tout "l'intérêt d'analyser un film atypique» (Larouche 1991b, p. 14) comme Kalamazoo, d'André Forcier, c'est que, dégageant l'axe d'orientation de l'œuvre, l'analyse descriptive ne révèle pas les insuffisances de certains modèles théoriques sans affirmer, par cela même, les points de singularité qui composent le film. Mais, pour conserver sa consistance et sa cohérence, l'analyse descriptive devra trouver le moyen d'agencer ses limites: on ne recourra à la syntagmatique de Metz que si la limite qu'elle atteint peut elle-même être explorée par un autre modèle d'analyse qui, à son tour, devra déboucher sur une limite, toutes les limites convergeant vers des processus de "glissements sémantiques", des "stratégies discursives" qui font éclater le discours, des "transformations" entraînant "un changement continuel de points de vue» (p. 14-17), aucun des modèles, ni le lieu de convergence des limites, ne constituant une théorie unifiée ni une méthode générale, la consistance et la cohérence de l'analyse ne relevant désormais que de cette forme de répétition et de la forme d'engagement de l'interprète - sa posture éthique, dont il faudra décidément découvrir la nature.

Ce mode de ré-enchaînement des limites de chacun des modèles d'analyse explique sans doute en partie l'extrême discrétion avec laquelle Michel Larouche convoque les systèmes théoriques. On ne peut pas dire de lui qu'il appartient à la sémiologie linguistique, à la narratologie, à la sémiopragmatique, aux théories de la réception, etc.; car, si ces théories sont invoquées, ce n'est précisément pas comme système. S'il leur emprunte un concept ou un outil, une hypothèse ou un problème, ce n'est ni pour les éprouver ni pour les étendre. Que chaque modèle théorique atteigne à une limite, 
ce n'est pas pour lui l'occasion de vérifier les modèles, c'est-àdire de leur adjoindre une nouvelle procédure ou un nouveau concept pour qu'ils se remettent à fonctionner, mais l'occasion de respirer un instant, tout seul, dans le vide que laisse l'inadéquation de la théorie à l'objet: il n'est jamais plus heureux que lorsque la mécanique se casse. Mais pourquoi? D’une part, Michel Larouche donne l'impression de convoquer la théorie à son corps défendant, comme contraint à une obligation, comme s'il cédait à une pression institutionnelle. Aussi semble-t-il éprouver un soulagement quand, reprenant toujours la même citation de l'un ou de l'autre des maîtres du moment, il parvient à lui faire jouer le rôle d'un mot de passe, d'une formule mondaine (à l'exception très certainement d'une citation dont on parlera). D'autre part, il est d'autant plus légitimé de reprendre toujours les mêmes formules théoriques qu'elles n'ont pas chez lui pour fonction d'expliquer le texte sur lequel elles portent; ce sont plutôt elles qui seront expliquées par le texte, d'où l'intérêt de les reprendre pour leur faire produire de nouveaux effets de sens. Plus précisément, chez lui, la théorie n'explique pas un film sans rendre des comptes; elle n'explique pas sans s'expliquer elle-même, et elle trouve une nouvelle explication à chaque nouvelle rencontre avec un film: c'est en dépliant un scénario ou en arpentant un dispositif, en affrontant chacun des points de singularité d'un texte plurifilmique ou d'une nouvelle technologie de la représentation qu'une théorie s'explique, c'est-à-dire déplie jusqu'au bout la série de ses problèmes, se compose concept par concept, contourne ses apories ou bute contre elles, s'effondre ou s'ouvre à partir de ses limites, etc. Un film passe par un point de singularité, l'image s'immobilise, et c'est alors la théorie narratologique qui est forcée de s'expliquer sur le travail du signifiant et de "reconsidérer le concept de focalisation" (Larouche 1988, p. 137). Le dispositif cinématographique tout entier passe par une mutation technologique, et ce sera l'occasion «d'un déplacement certain [...] des données les plus fondamentales au sein de la théorie cinématographique» (Larouche 1991c, p. 86).

L'analyse descriptive pratiquée par Michel Larouche comprend donc une forme de répétition: multiplier les approches, 
cela signifie que l'analyse d'une série d'éléments sera nécessairement reprise par une autre analyse qui, repartant chaque fois à zéro, va recombiner autrement ces éléments, les retrouver sous un nouvel angle, sous une nouvelle forme, à un autre niveau. Quiconque voudra s'en convaincre relira la minutieuse analyse descriptive du film So Is This, de Michael Snow. À chaque étape de l'analyse, le même matériel est sans cesse repris pour être soumis à une nouvelle répartition, jusqu'à atteindre une limite:

Déterminer le nombre de plans dans SO IS THIS apparaît dès lors comme une opération insensée que l'analyste se propose quand même d'entreprendre, espérant peut-être pouvoir y déployer une grande syntagmatique? Jusqu'à la reprise d'une partie du film à une très grande vitesse, il y a 1201 mots auxquels il faut ajouter le nombre équivalent d'intervalles, ce qui nous conduit à 2402 plans. Par la suite il n'y a pas véritablement d'intervalles et lorsqu'ils apparaissent, ils occupent la place des mots manquants. Si on ajoute donc encore 1201 plans, on obtient le chiffre de 3603 . La suite du film comporte 661 mots [...], ce qui produit avec les intervalles, 1322 nouveaux plans. Nous obtenons donc le nouveau chiffre de 4925 . À ces plans il faudrait ajouter la lumière blanche qui tient la place d'un mot, et aussi son intervalle, à la suite des mots isolés, ce qui nous reporte à 4927 plans. Il faudrait aussi préciser que les mots «held", «repeated" et "filmed » sont entrecoupés de cartons noirs, ce qui crée un certain clignotement et multiplie en conséquence le nombre de plans, qu'après la fin du texte des cartons colorés, dont il est impossible de déterminer l'équivalence en plans, ont remplacé les mots, que l'amorce fait partie du film, etc. S'il n'y a pas de syntagmatique possible de SO IS THIS, c'est parce que le film ne fonctionne pas à partir du plan (Larouche 1984a, p. 35).

Me croit-on quand je dis de Michel Larouche qu'il est un grand comique! Sa répétition est toujours fondamentalement ironique, elle lui permet de remonter de la loi (la syntagmatique, par exemple) à son principe (le découpage), lequel s'oppose en tous points aux exigences et au règne de cette loi parce que le film et l'analyse en auront observé et appliqué l'essentiel paradoxe (à force de découper, il n'y a plus de syntagme): du Beckett. À chaque étape de l'analyse, le même matériel est donc repris et réparti autrement, jusqu’à atteindre la limite d'un renversement. C'est cette limite qui fait déboucher 
chaque fois sur une seconde répétition : c'est maintenant d'étape en étape que le matériel est sans cesse repris pour être soumis à une nouvelle perspective; ce qu'il aura compté et recompté, Michel Larouche va alors en analyser l'effet performatif (1984a, p. 34), la logique combinatoire (p. 33), la stratégie discursive (p. 32). De parcours en parcours, il répète, et chacun de ses parcours est en soi répétitif, si bien que les résultats ne s'additionnent pas pour saturer l'espace d'interprétations, mais se reprennent suivant une distance indécomposable. Les répétitions se reprennent les unes les autres: qu'est-ce que cela veut dire? D'une part, une répétition ne répète jamais à l'identique, mais en différenciant: après avoir compté les mots, on compte ce qui les sépare, on compte ce qui les remplace, on compte ce qui les fragmente, avant de faire le compte de ce qui ne se laisse pas dénombrer. D’autre part, une répétition ne répète pas sans impliquer en elle toutes les autres répétitions, qui elles aussi l'impliquent, l'impliquaient ou l'impliqueront: le découpage irrationnel des phrases et des mots implique la stratégie discursive («la pratique généralisée du mensonge» [Larouche 1984a, p. 32]), la logique combinatoire (un «nouveau rapport du mot à l'espace» [p. 33]), un effet performatif (une énonciation «toujours en excès sur l'énoncé» [p. 34]), et inversement. En reprenant en elle toutes les répétitions, mais sous un autre mode ou à un autre niveau, chaque reprise de l'analyse ouvre une perspective sur elles toutes. Du livre sur Jodorowsky jusqu'à l'analyse de Kalamazoo en passant par celle de So Is This, Michel Larouche a suivi cette logique de l'analyse descriptive, a pratiqué cette répétition à distance ou par implication. Et il retrouvera bientôt cette logique au coeur de l'adaptation, du transcodage ou de la transmédiatisation - «Le scénario, en réfractant la nouvelle dont il s'inspire, permet de réfléchir le caractère cinématographique de l'écriture de Gabrielle Roy au sein même de sa nouvelle» (Larouche 1997b, p. 10) —, avant qu'elle ne devienne la logique d'enchainement de ses propres textes sur l'effet-photo (Larouche 1988; 1989; 1991d) ou sur l'adaptation (1997a; 1997b; 1997c; Larouche et Cardinal 2003).

Si les textes filmiques ou médiatiques peuvent faire penser la théorie plutôt que de se laisser expliquer par elle, et si plus 
profondément la théorie n'explique pas ces textes sans se voir forcée de s'expliquer elle-même, c'est que les textes qui intéressent Michel Larouche fonctionnent eux-mêmes par répétition et par implication. Les textes et l'analyse ont une même puissance de pensée, qu'ils actualisent selon leurs moyens propres: ils réutilisent pour comprendre (Larouche 1985b, p. 49). La combinatoire est devenue une forme de pensée, qui comprend un choix d'éléments, une combinaison et une recombinaison perpétuelle de ces éléments, avec des effets de détournement, de renversement, de dénonciation, de caricature, de parodie, etc. (1985b, p. 37-54). Quand Jodorowsky reprend les éléments mythiques du western, ce n'est pas sans les recombiner suivant une logique de la bande dessinée qui, "alliée à un système théâtral excessivement développé", va produire un effet d'abstraction entraînant le cinéma vers le théâtre de marionnettes (1985b, p. 83). Quand Sarah Maldoror choisit de tisser son film Sambizanga (1972) de répétitions, ce n'est pas sans inscrire au cœur de chaque reprise un léger décalage, "permettant à l'humour, à la violence contenue, à la sentimentalité, à la sensibilité de s'exprimer librement", ce qui a pour effet de redonner aux personnages emprisonnés, persécutés ou disparus, "le droit de vivre décemment" (Larouche 1991a, p. 31-32). De même, l'écriture de la critique "est profondément marquée par un processus d'adaptation", considéré comme une "zone de création" ou de recombinaison des images et des sons (Larouche et Cardinal 1996b, p. 131), avec pour effet de «relancer chez le lecteur le travail d'appropriation d'une œuvre et de production de sens» (Larouche 1997a, p. 32). De même, l'analyse d'un film ne combine pas des modèles théoriques sans recombiner leurs limites, avec pour effet "de préciser où exactement se situe la singularité de l'œuvre» (Larouche 1991b, p. 14). Bref, si «on rejette l'empirisme philosophique et qu'on admet des niveaux d'intelligibilité, tout un réseau de corrélations se tisse à travers le film, à l'égard duquel on peut dès lors parler de théorie" (Larouche 1985b, p. 36). Inversement, si on comprend les répétitions et les implications du texte théorique comme autant de formes de réflexivité, d'autoreprésentation et d'autoréférence, on pourra dire qu'il participe d'une modernité de l'écriture qui 
rend floues les frontières entre théorie et cinéma (Larouche et Cardinal 1996b, p. 136). Les ressemblances externes, avec les mouvements de réversibilité qu'elles déclenchent, trouvent donc leur raison dans cette forme de pensée commune au texte et à l'analyse du texte. C'est dire que la répétition et l'implication des répétitions ne circulent pas du texte à l'analyse comme des procédures constituées que l'une et l'autre pourraient réciproquement s'emprunter ou simplement réfléchir. En d'autres termes, si Michel Larouche s'intéresse au cinéma expérimental, au cinéma atypique ou à l'adaptation, ce n'est pas parce que, fonctionnant tous à coups de répétitions, il lui serait plus facile d'appliquer sur ces objets ses propres analyses répétitives. C'est tout autre chose qui est ici en jeu : si ces films et Michel Larouche répètent, c'est que lui et eux sont occupés à remonter jusqu'aux conditions de la perception et de la pensée. Tandis que ces films deviennent "le lieu de coexistence de modalités perceptuelles diverses en puissance», tandis que l'expérience du spectateur produit elle-même "les conditions de perception" (Larouche 1995 d, p. 142), il revient au théoricien «de développer de nouveaux cadres conceptuels" (Larouche 1991b, p. 21). Si le film, en tissant un réseau de répétitions, fait de la théorie, c'est qu'il est ainsi le récit de sa production de sens. Si l'effet-photo est une "surcharge figurative" et un "aveu de montage" qui désignent "le processus narratif lui-même», c'est qu'il transforme en même temps tout le film en un récit de genèse des sens et du sens, c'est qu' «il entraîne une appréhension conceptuelle de l'espace et du temps » (Larouche 1988, p. 135-136). «Les films qui produisent l'effet photographie redoublent les mouvements psychiques qui agitent habituellement le spectateur»(p. 135-136). Quand les rapports de l'image et du son aboutissent tous à une "dé-hiérarchisation systématique des codes du cinéma narratif classique» tout en produisant "un véritable montage audio-visuel», ils participent de facto à un "déchiffrement de l'expérience filmique" (Larouche 1985a, p. 69). Inversement, si la théorie ou l'analyse descriptive pratiquent une forme d'esthétique transcendantale, c'est que tantôt elles remontent jusqu'au grain et à la texture de l'image comme vers l'émergence d'un nouvel usage des sens 
(Larouche 1985b, p. 33), et tantôt elles suivent les différences entre des modes et des moyens de représentation comme autant de modalités de perception, orientant le point de vue de l'analyse intertextuelle ou intermédiatique "en fonction de l'étude des affects propres à chacun des arts" (Larouche 1991d, p. 62). Et si elles s'intéressent encore au mode énergétique du cinéma, c'est que, par modulation du tempo et de l'intensité des images et des sons, ce mode énergétique interpelle le spectateur; plus, il l'entraîne dans des vibrations où il naît «à lui-même et à un monde» (Larouche 1999, p. 106).

Ces conditions de la perception et de la pensée, Michel Larouche les trouve en des points de singularité, c'est-à-dire précisément là où le texte filmique ou médiatique rencontre un problème de consistance ou de cohérence, cependant que la théorie trouve sa limite. Tous ses travaux de recherche consistent essentiellement à découvrir et à explorer ces points de singularité, comme on l'entend en physique d'un point de congélation, d'ébullition, de cristallisation. Un point de singularité, ce sera un point de "rencontre entre le cinéma et la réalité africaine», qui va entraîner l'homogénéité des espaces du cinéma classique dans "un système d'ubiquité nouveau " (Larouche 1991a, p. 35). Un point de singularité, ce sera un point de renversement et d'enveloppement du fixe et du mouvant, qui produira un nouveau type d'images-mouvement, auxquelles " on ne peut attribuer de façon systématique les propriétés de la photographie [...], en même temps qu'elles different des images mouvantes auxquelles le cinéma nous a habitués" (Larouche 1988, p. 134). Un point de singularité, ce sera un point d'hybridité entre l'analogique et le numérique, qui rend indistincte la frontière entre la trace et le programme tout en participant d'un «déplacement perceptuel ", celui de la "vraisemblance visuelle" à " un réalisme abstrait" (Larouche 1991c, p. 86; voir aussi 1999, p. 104). Un point de singularité, ce sera un point de transcodage, le lieu d'un choc entre une forme d'écriture et une autre, qui place l'avenir médiatique d'un dialogue, d'un personnage, d'une voix narrative, «sous le signe de la rupture, de la fracture, redoublant le processus de mutation lié à toute entreprise d'adaptation" (Larouche et Gauvin 1995b, p. 89). 
Tous ces points de singularité, et d'autres encore, ce sont eux qui entraînent les textes filmiques et médiatiques dans le régime de répétition qui leur est propre, ce sont eux qui déclenchent le processus de combinaison et de recombinaison, vaine tentative pour épuiser le potentiel du métissage, de l'hybride, du "vacillement", de la "tension", etc., mais dont la vanité produit la multiplication des séries qui constituent le film, tandis que l'analyse descriptive doit se résoudre à multiplier les approches, construisant à son tour un espace intermédiaire où rejouer ces points de singularité avec des points ordinaires, de façon à produire du sens :

espace de rencontre; espace de circulation du savoir; mais aussi espace intermédiaire comme on dit état intermédiaire, mixte, hybride: un espace construit à partir de l'entrelacement de l'activité imaginaire et de l'activité de perception (Larouche et Cardinal 1996b, p. 132).

Mais si tous ces points de singularité restent inépuisables ou irréductibles, c'est que se tient en leur fond une altérité radicale, qui anime et le texte filmique et l'analyse descriptive tout en restant elle-même invisible ou indicible. Cet invisible pèse sur tous les films qui intéressent Michel Larouche, tous menacent de s'effondrer sur eux-mêmes; d'où le formidable effort du théoricien, effort de description et d'interprétation par lequel ils redeviennent nécessaires et vitaux. Mais celui qui fait cet effort affirme en même temps une profonde et cruelle modestie, profonde parce qu'elle affirme la différence qu'aucune répétition ne pourra jamais ramener au même, cruelle parce qu'elle le condamne au silence. Une citation va hanter plusieurs des textes de Michel Larouche, servant presque toujours de conclusion, sous la forme d'un retour sur les efforts d'analyse qu'il vient de fournir et sur la pure différence que constitue toujours le texte qui était soumis à l'examen. Cette citation n'a plus rien du mot de passe, de la formule mondaine qui lui permettait de ne pas trop s'afficher ou de rester le plus discret possible; au contraire, il semble alors que, reprenant les mots d'un autre, il s'expose tout entier:

L'altérité n'est pas représentable car l'identification à l'autre ne peut se faire qu'à travers mes propres modèles discursifs qui, eux- 
mêmes, ont été produits pour exprimer ce que je sais, ce que je suis ou ce que j'imagine et n'ont été produits que pour cela; d'où leur incapacité à rendre compte de tout ce qui est à l'extérieur de moi et de mon univers (Edmond Cros, cité dans Larouche 1995c, p. 232 ; 1996a, p. 156 ; 1999, p. 108; Larouche et Cardinal 1995a, p. 14; Larouche et Gauvin 1995b, p. 92).

Mais qu'est-ce qui toujours ramène Michel Larouche à sa table de travail, en dépit de cette altérité radicale sur laquelle viendraient rebondir ses tentatives d'analyse descriptive? La réponse doit répondre en même temps à cette autre question que nous avons laissée en suspens: quelle posture éthique donne à ses analyses le pouvoir de tenir toutes seules, c'est-à-dire de conserver leur consistance et leur cohérence, malgré leur vocabulaire daté ou leur objet disparu, tout en relançant chez le lecteur «le travail d'appropriation d'une ouvre et de production de sens» (1997a, p. 32) ? La posture éthique de Michel Larouche a en commun avec les films qui l'ont intéressé (films surréalistes, expérimentaux, atypiques) un même problème et une même visée: un monde dont il faudrait s'échapper ou qu'il faudrait changer, qu'on ne pourra changer qu'en s'échappant de nos schèmes mentaux, de nos schèmes de perception (Larouche 1985b, p. 34). S’il se méfie des théories universalisantes (1984a, p. $36 ; 1985 a$, p. 68 et $70 ; 1991$ b, p. 13), s'il ne reprend discrètement que des morceaux, des mouvements, des gestes théoriques, s'il ne le fait qu'en cherchant d'emblée leurs limites et en ne se laissant prendre à aucune, c'est qu'il ne s'agit pas seulement pour lui d'analyser un film et de le comprendre: il s'agit surtout de décrire et d'analyser des mutations, dans l'espoir de changer lui-même par cette observation. Si l'analyse descriptive est un instrument capable d'engendrer une richesse infinie de significations, cette richesse n'aura de sens, comme les films auxquels elle répond et qu'elle prolonge, qu'à condition «de réconcilier tout homme, momentanément ou non, avec l'idée de vie» (André Breton, cité dans Larouche 1984b, p. 154), qu’à condition de redonner le droit de vivre décemment à un peuple (Larouche 1991a, p. 32), de rechercher de nouvelles lois pour 
un nouveau monde (1985b, p. 171). Comme peu de théoriciens du cinéma, mais comme les cinémas qu'il a aimés, Michel Larouche "essaie de lier la connaissance, l'acte de la connaissance, les conditions de cet acte de connaissance et ses effets, à une transformation dans l'être même du sujet " (Foucault 2001, p. 30).

\section{Université de Montréal}

\section{RÉFÉRENCES BIBLIOGRAPHIQUES}

Bazin 1985: André Bazin, "Ontologie de l'image photographique», Qu'est-ce que le cinéma?, Paris, Cerf, 1985, p. 9-17.

Foucault 2001 : Michel Foucault, L'Herméneutique du sujet. Cours au Collège de France, 1981-1982, Paris, Gallimard/Seuil, 2001.

Larouche 1984a : Michel Larouche, "SO IS THIS : la transgression des théories", Les Dossiers de la Cinémathèque, $\mathrm{n}^{\circ} 12$, 1984, p. 32-36.

Larouche 1984b: Michel Larouche, "Surréalisme cinématographique au Québec", dans Luis de Moura Sobral (dir.), Surréalisme périphérique, Montréal, Université de Montréal, Département d'histoire de l'art, 1984, p. 147-157.

Larouche 1985a: Michel Larouche, "Vers la symbiose image-son dans le cinéma expérimental: une utopie?». Protée, vol. 13, n 2, 1985, p. 67-71.

Larouche 1985b: Michel Larouche, Alexandre Jodorowsky. Cinéaste panique, Paris, Albatros, 1985.

Larouche 1986: Michel Larouche, "La place singulière du cinéma expérimental», Dérives, $\mathrm{n}^{\circ}$ 52, 1986, p. 51-62.

Larouche 1988 : Michel Larouche, "Les enjeux de l'"effet photographique" au cinéma ", Protée, vol. 16, nos 1-2, 1988, p. 134-138.

Larouche 1989: Michel Larouche, L'effet photographique": problèmes de représentation et de narration, Montréal, Cinémathèque québécoise-AQEC, 1989.

Larouche 1991a: Michel Larouche, "Le temps que l'on met à marcher», Films d'Afrique, Montréal, Guernica, 1991, p. 21-38.

Larouche 1991b: Michel Larouche, "Kalamazoo d'André Forcier ou l'intérêt d'analyser un film atypique", dans Jacqueline Viswanathan (dir.), Cinéma français et québécois. Perspectives et méthodes d'approche, Burnaby, APFUCC, 1991, p. 13-23.

Larouche 1991c: Michel Larouche, "Nouvelles technologies et troisième dimension", Cinémas, vol. 1, n 3, 1991, p. 77-87.

Larouche 1991d: Michel Larouche, "Effet-photo" et nouvelles images: vers un cinéma de l'affect", Protée, vol. 19, n³ 3, 1991, p. 61-65.

Larouche 1993: Michel Larouche, "La loi du genre», CinémAction, n 68, 1993, p. 204-207.

Larouche 1995c: Michel Larouche, "Genre et transgression au sein de la réception critique française des films québécois ", dans Ginette Adamson et Jean-Marc Gouanvic (dir.), Francophonie plurielle, Montréal, Hurtubise HMH, 1995, p. 227-235.

Larouche 1995d: Michel Larouche, "La représentation de Montréal dans les images immobilisées du cinéma québécois ", Tangence, n 48, 1995, p. 136-144. 
Larouche 1996a: Michel Larouche, "Pierre Perrault et la "parlure" du Québec», L'aventure du cinéma québécois en France, Montréal, XYZ éditeur, 1996, p. 145-157.

Larouche et Cardinal 1996b: Michel Larouche et Serge Cardinal, «L'écriture de la critique", Cinémas, vol. 6, nos 2-3, 1996, p. 127-139.

Larouche 1997a: Michel Larouche, "L'adaptation filmique», Protée, vol. 25, n 1, 1997, p. 29-35.

Larouche 1997b: Michel Larouche, "Le vieillard et l'enfant: le scénario de Gabrielle Roy", Cahiers franco-canadiens de l'Ouest, vol. 9, no 1-2, 1997, p. 3-17.

Larouche 1997c: Michel Larouche, "Le vieillard et l'enfant de Gabrielle Roy: de la nouvelle au film ", dans Gilles Dupuis, Carla Frata et Manon Riopel (dir.), Littérature et cinéma du Québec, Rome, Bulzoni Editore, 1997, p. 37-48.

Larouche 1999: Michel Larouche, «Les nouvelles technologies au cinéma: le retour du mode énergétique?», dans Michel Bouvier, Michel Larouche et Lucie Roy (dir.), Cinéma: acte et présence, Québec/Lyon, Nota bene/Centre Jacques Cartier, 1999, p. 99-110.

Larouche 2001 : Michel Larouche, «Imaginaire américain et cinéma québécois », dans Jaap Linvelt et François Paré (dir.), Frontières flottantes/Shifting Boundaries, Amsterdam/New York, Éditions Rodopi, 2001, p. 245-253.

Larouche et Cardinal 1995a: Michel Larouche et Serge Cardinal, "Une écriture "scénarique" ", dans Paul Warren (dir.), Zola et le cinéma, Ste-Foy/Paris, Presses de l'Université Laval/Presses de la Sorbonne Nouvelle, 1995, p. 3-16.

Larouche et Cardinal 2003 : Michel Larouche et Serge Cardinal, «Le scénario : points de vue", dans Michel Larouche (dir.), Cinéma et littérature au Québec: rencontres médiatiques, Montréal, XYZ éditeur, 2003, p. 13-25.

Larouche et Gauvin 1995b : Michel Larouche et Lise Gauvin, «Laventure ambiguë: de la parole romanesque au récit filmique», Études françaises, vol. 31, n 1, 1995, p. $85-93$.

\begin{abstract}
The Last Modest Scholar Serge Cardinal

Michel Larouche, the co-founder of the journal Cinémas, taught in the Dépatement d'histoire de l'art et d'études cinématographiques at the Université de Montréal until 2005. His work introduced us to little-known types of cinema (such as experimental and African cinema), unknown cinema (the films of Jodorowsky and independent Quebec cinema) and hybrid cinema (films which use photography, new technology or literature). But what are the methods, problems and concepts Michel Larouche has bequeathed us? In particular, what intellectual ethic has he modestly left to film studies?
\end{abstract}

